\title{
Apocynin Ameliorates Pressure Overload-Induced Cardiac Remodeling by Inhibiting Oxidative Stress and Apoptosis
}

\author{
J.-J. LIU ${ }^{1^{*}}$, Y. LU ${ }^{2 *}$, N.-N. PING3, X. LI ${ }^{1}$, Y.-X. LIN ${ }^{1}$, C.-F. LI ${ }^{4}$ \\ "These authors contributed equally to this work as first authors.
}

${ }^{1}$ Department of Physiology and Pathophysiology, Xi'an Jiaotong University Health Science Center, Xi'an, Shaanxi Province, China, ${ }^{2}$ Department of Pharmacology, Xi'an Jiaotong University Health Science Center, Xi'an, Shaanxi Province, China, ${ }^{3}$ Shaanxi Blood Center, Xi'an, Shaanxi Province, China, ${ }^{4}$ Department of Obstetrics and Gynecology, The First Affiliated Hospital of Xi'an Jiaotong University Health Science Center, Xi'an, Shaanxi Province, China

Received November 10, 2015

Accepted June 21, 2016

On-line October 26, 2016

\section{Summary}

Oxidative stress plays an important role in pressure overloadinduced cardiac remodeling. The purpose of this study was to determine whether apocynin, a nicotinamide adenine dinucleotide phosphate (NADPH) oxidase inhibitor, attenuates pressure overload-induced cardiac remodeling in rats. After abdominal aorta constriction, the surviving rats were randomly divided into four groups: sham group, abdominal aorta constriction group, apocynin group, captopril group. Left ventricular pathological changes were studied using Masson's trichrome staining. Metalloproteinase-2 (MMP-2) levels in the left ventricle were analyzed by western blot and gelatin zymography. Oxidative stress and apoptotic index were also examined in cardiomyocytes using dihydroethidium and terminal deoxynucleotidyl transferase dUTP nick end labeling (TUNEL), respectively. Our results showed that abdominal aorta constriction significantly caused excess collagen deposition and cardiac insult. Treatment with apocynin significantly inhibited deposition of collagen and reduced the level of MMP-2. Furthermore, apocynin also decreased the NADPH oxidase activity, reactive oxygen species production and cardiomyocyte apoptotic index. Interestingly, apocynin only inhibited NADPH oxidase activity without affecting its expression or the level of angiotension II in the left ventricle. In conclusion, apocynin reduced collagen deposition, oxidative stress, and inhibited apoptosis, ultimately ameliorating cardiac remodeling by mechanisms that are independent of the renin-angiotensin system.

\section{Key words}

Apocynin - Cardiac remodeling • Angiotension II - NADPH oxidase • Reactive oxygen species • Apoptosis.

\section{Corresponding author}

C.-F. Li, Department of Obstetrics and Gynecology, The First Affiliated Hospital of Xi'an Jiaotong University Health Science Center, Xi'an 710061, Shaanxi Province, China. Fax: +86 29 82657497. E-mail: lichunfang626@126.com

\section{Introduction}

Heart failure (HF) is the end stage of various kinds of cardiovascular diseases (CVD), which is a leading cause of morbidity and mortality in middle age and aged people (Greenberg et al. 2012, Al Suwaidi et al. 2012). Cardiac remodeling has been suggested to be associated with poor prognosis in patients with cardiovascular disorders (Cohn et al. 2000, Gonzalez et al. 2011). Cardiomyocyte hypertrophy, apoptosis, and subsequent fibroblast proliferation play critical roles in the process of cardiac remodeling, which ultimately accelerates cardiac fibrosis and contributes to cardiac dysfunction (Hassan et al. 2012, Mitra et al. 2013).

The renin-angiotensin system (RAS) plays an important role in the cardiac remodeling process and is involved in the pathogenesis of HF (Nagalingam et al. 2013). Growing evidence confirms that activation of the 
RAS leads to a large generation of reactive oxygen species (ROS) that contribute to cardiomyocyte hypertrophy, apoptosis, and fibrosis in cardiac remodeling (Choudhary et al. 2008). Captopril, as the earliest angiotensin converting enzyme inhibitor (ACEI), exerts cardioprotective effects by increasing tissue antioxidant activity, scavenging different types of ROS, and then decreasing the deposition of collagen, inflammatory response and myocyte apoptosis, which ultimately ameliorating cardiac remodeling (St John Sutton et al. 1997, Li et al. 2015). Recent studies showed that ROS are produced by mitochondria, xanthine oxidases, cytochrome P450 reductase, and nicotinamide adenine dinucleotide phosphate (NADPH) oxidase (Bedard and Krause 2007). Among these, NADPH oxidase is a primary source for ROS production at the presentation of CVD. NADPH oxidase contains phox units $\left(\mathrm{gp} 91^{\text {phox }}, \mathrm{p} 22^{\text {phox }}, \mathrm{p} 40^{\text {phox }}\right.$, $\mathrm{p} 47^{\text {phox }}, \mathrm{p} 67^{\text {phox }}$ ) and the small GTPase (Rac1 or Rac2) (Cai et al. 2003, Jin et al. 2008, Lassegue and Griendling 2010). ROS derived from NADPH oxidase play a vital role in pathophysiological conditions such as atrial fibrillation, myocardial infarction, and hypertension (Liu et al. 2012b, Al-Rasheed et al. 2013, Cheng et al. 2014). Therefore, inhibition of this enzyme may be an attractive and potential therapeutic target for the treatment of HF (Schwarzer et al. 2014).

Apocynin (4-hydroxy-3-methoxy-acetophenone), a constituent of the Himalayan herb Picrorhiza kurrooa Royle (Scrophulariaceae) has been used as an inhibitor of NADPH oxidase (Engels et al. 1992, Babior et al. 2002). Recent studies showed that apocynin can improve atrial fibrillation in hearts with infarction, and also has antihypertensive effects on spontaneously hypertensive rats (Sovari et al. 2008, Ciarcia et al. 2010). We previously reported that apocynin ameliorated pressure overloadinduced cardiac hypertrophy by reducing ROS production (Liu et al. 2010). However, it is not clear whether apocynin can attenuate cardiac remodeling in rats with pressure overload. Therefore, in the present study we investigated whether apocynin might be able to attenuate cardiac remodeling in Sprague-Dawley (SD) rats with pressure overload. We also further explored the possible mechanisms of action of apocynin for suppressing cardiac fibrosis.

\section{Material and Methods}

Animals

Adult male SD rats (8-10 week-old) used in this study, were supplied by the Experimental Animal Centre of Xi'an Jiaotong University. The animals were housed under controlled temperatures $\left(20 \pm 5^{\circ} \mathrm{C}\right)$, humidity (60-75\%), and light/dark cycles (12 h day/12 h night). All experimental procedures were in compliance with the Guide for the Care and Use of Laboratory Animals published by the National Institutes of Health (NIH publication No. 85-23, revised 1996). The animal protocols were approved by the Animal Care and Use Committees of Xi'an Jiaotong University.

\section{Induction of cardiac remodeling via abdominal aorta constriction and drug administration}

Abdominal aorta constriction was performed as described previously (Chen et al. 2010). Briefly, after being fasted for $12 \mathrm{~h}$, rats $(\mathrm{n}=50)$ were anesthetized with pentobarbital sodium $(45 \mathrm{mg} / \mathrm{kg})$ by intraperitoneal injection, and the aorta was dissected above the two renal arteries. A silver clip ( $0.70 \mathrm{~mm}$ internal diameter) was placed on the aorta abdominalis above the level of the left renal arteries. 7 days after the surgical procedure, rats were randomly divided into four groups: abdominal aorta constriction group (AAC, $\mathrm{n}=7$ ), apocynin (Apo) + AAC group (Apo, $200 \mathrm{mg} / \mathrm{kg} /$ day, $\mathrm{n}=8$ ), captopril (Cap) + AAC group (Cap, $75 \mathrm{mg} / \mathrm{kg} /$ day, $\mathrm{n}=8$ ). The Sham group $(n=10)$ was subjected to the same surgical procedure without clip placement. All drugs were purchased from Sigma (St Louis, MO, USA) and dissolved in drinking water. The animals were sacrificed 8 weeks after surgery and the hearts were collected for histological analysis (Fig. 1).

\section{Hemodynamic data analysis}

To examine the effect of Apo on cardiac function, we measured hemodynamics 8 week after abdominal aorta constriction. Animals were anesthetized with pentobarbital sodium $(45 \mathrm{mg} / \mathrm{kg})$ by intraperitoneal injection, and the right carotid arterial connected to pressure transducers was introduced into the left ventricle to measure heart rate (HR), systolic arterial pressure (SAP), left ventricular systolic pressure (LVSP), left ventricular end-diastolic pressure (LVEDP) and maximum/minimum values of the first derivative of left ventricular pressure $\left(\mathrm{dp} / \mathrm{dt}_{\max }\right.$ and $\left.\mathrm{dp} / \mathrm{dt}_{\min }\right)$. All data were recorded with PowerLab (AD Instruments) in all groups under a 30-minute steadystate condition.

\section{Blood sampling and tissue preparation}

At the eighth week, all rats were anesthetized 
with pentobarbital sodium by intraperitoneal injection. Blood was drawn from the abdominal aorta, allowed to clot, centrifuged at $5,000 \mathrm{x} \mathrm{g}$ for $10 \mathrm{~min}$ and the serum was stored at $-80^{\circ} \mathrm{C}$ for further analysis. Next, the whole hearts from the rats were quickly removed, washed with cold phosphate buffered saline $(2.7 \mathrm{mM} \mathrm{KCl}, 137 \mathrm{mM}$

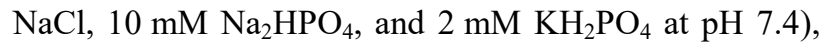

and sliced transversely from apex to base freeing the right ventricular wall. The left ventricular wall was snapfrozen in liquid nitrogen and store at $-80^{\circ} \mathrm{C}$. The angiotensin II (Ang II) activity (concentration) in tissue and serum was analyzed using a commercial kit (Dong Ya Radioimmunology Institute, Beijing, China) following the manufacturer's instructions.

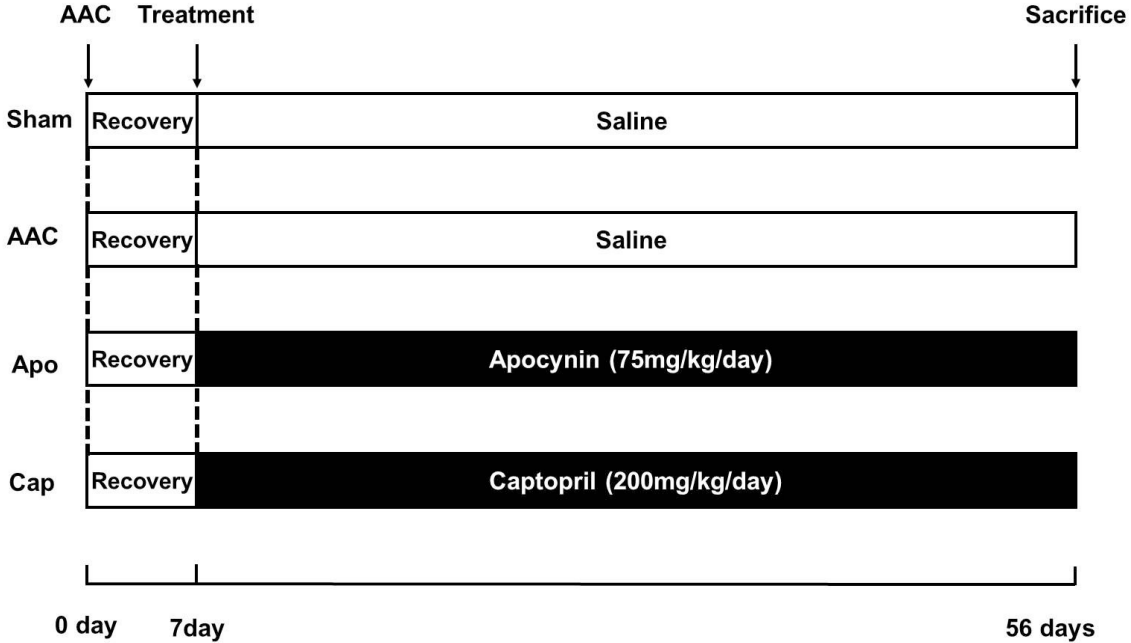

Fig. 1. The experimental groups used in the study. Rats $(n=40)$ were anesthetized with pentobarbital sodium $(45 \mathrm{mg} / \mathrm{kg})$ by intraperitoneal injection, and a silver clip $(0.70 \mathrm{~mm}$ internal diameter) was placed on the aorta abdominalis above the level of the left renal arteries. 7 days after the surgical procedure, rats were randomly divided into four groups: Sham, rats without abdominal aorta constriction; AAC, rats with abdominal aorta constriction; Apo, AAC rats treated with apocynin; Cap, AAC rats treated with captopril. The animals were sacrificed 8 weeks after surgery and the hearts were collected for histological analysis.
Collagen deposition determination by Masson's trichrome staining

Masson's trichrome staining was performed in the rat hearts to determine collagen deposition. Hearts were excised, washed in PBS at $4{ }^{\circ} \mathrm{C}$, cut into 3 transverse sections close to the apex to visualize the left and right ventricles, and then fixed in $4 \%$ formalin by paraffin embedding. They were sectioned $(5 \mu \mathrm{m})$ and stained. Cardiac fibrosis was analyzed by morphometry via Masson's staining and visualized by light microscopy.

\section{Assessment of oxidative stress}

Myocardial superoxide anion production was measured using the fluorescent dye, dihydroethidium (DHE; Beyotime Institute of Biotechnology, Nanjing, China) as described previously.(Courtois et al. 2003) Briefly, $5 \mu \mathrm{m}$ fresh frozen sections from the hearts were incubated in DHE $(10 \mu \mathrm{M})$ for $30 \mathrm{~min}$ at $37^{\circ} \mathrm{C}$ and photographed under fluorescent microscopy. The red fluorescence intensity in sections was analyzed using the Image-Pro Plus 6.0 (Media Cybernetics, Silver Spring, MD).

Heart tissue was homogenized in ice cold PBS with a homogenizer to yield a $10 \%$ (wt/vol) myocardial homogenate. According to the manufacturer's instructions, Malondialdehyde (MDA) level, superoxide dismutase (SOD) and NADPH oxidase activity (Bio-Rad
Laboratories, Hercules, CA, USA) were colorimetrically analyzed using a microplate spectrophotometer at $455 \mathrm{~nm}$, $500 \mathrm{~nm}$, and $550 \mathrm{~nm}$, respectively.

\section{Assessment of apoptosis}

To assess myocardial cell death or apoptosis, terminal deoxynucleotidyl transferase dUTP nick end labeling (TUNEL) assay was performed using a DNA fragmentation/fluorescence staining TUNEL apoptosis detection kit (Promega, Madison, WI, USA) according to the manufacturer's protocol. TUNEL positive cells were visualized by fluorescence microscopy (BX50, Olympus Co. Ltd., Tokyo, Japan) and the TUNEL index was calculated as the ratio of all groups compared to the Sham group.

Caspase-3 activity in myocardial tissue was evaluated using a microplate spectrophotometer at $405 \mathrm{~nm}$ by the biochemical reagent kit (Beyotime Institute of Biotechnology, Jiangsu, China).

\section{Real-time PCR}

Total RNA was extracted from left ventricular tissue using the TRIzol reagent (Invitrogen, Carlsbad, CA, USA) with a phenol-chloroform single step isolation. Reverse transcription was performed with an ExScript RT reagent kit (TaKaRa Bio, Shiga, Japan) according to 
the manufacturer's protocol. cDNA was diluted and amplified using random primers. The primer sequences for each gene were as follows:

$$
\text { p22 } 2^{\text {phox: }} \text { F-ATGGAGCGGTGTGGACAGAAG }
$$

and R-CGGACAGCAGTAAGTGGAGGAC; p67 $7^{\text {phox }}$ F-CCAGGCATTCCAAGATTGACAAGG and R-TGAACCACAGAGGCTACAACGC; $\beta$-actin: F-CTATCGGCAATGAGCGGTTCC and R-TGTGTTGGCATAGAGGTCTTTACG.

Relative RNA levels were expressed as the $\mathrm{p} 22^{\text {phox }} / \mathrm{p} 67^{\text {phox }}$ to $\beta$-actin ratio.

\section{Western blotting}

To examine the effects of apocynin on cardiac remodeling, levels of matrix metalloproteinase-2 (MMP-2), p22 $2^{\text {phox }}$ and $\mathrm{p} 67^{\text {phox }}$ were measured. Samples $(30 \mu \mathrm{g})$ were loaded onto $10 \%$ gradient SDS gels, separated by electrophoresis, and subsequently electroblotted onto a polyvinylidene difluoride (PVDF) membrane (Millipore, Billerica, MA, USA). After blocking with $5 \%$ BSA in Tris-buffered saline containing $0.1 \%$ Tween (TBST), the membrane was incubated with the following primary antibodies; MMP-2 (diluted 1:1,000, Bioworld technology Co., Ltd., Nanjing, China), p2 $2^{\text {phox }}$ (diluted 1:1,000, Cell Signaling Technology, Co., Ltd., Columbia, US), and p6 $7^{\text {phox }}$ (diluted 1:1,000, Cell Signaling Technology, Co, Ltd., Columbia, US) overnight at $4{ }^{\circ} \mathrm{C}$. Protein bands were detected using an ECL-Plus kit (PerkinElmer Life Science, Waltham, MA, USA) and quantified by densitometry using Quantity One software (Bio-Rad Laboratories, Berkeley, CA).

\section{Gelatin zymography}

Samples in a solution of $10 \%$ SDS, $20 \%$ glycerol, $0.5 \%$ bromophenol blue, and $0.125 \mathrm{mM}$ Tris- $\mathrm{HCl}(\mathrm{pH} 6.8)$ were loaded on $0.1 \%$ gelatin zymogram gels. Separating gels were washed twice for $30 \mathrm{~min}$ in $100 \mathrm{ml}$ of Triton X-100 (2.5\%) and incubated overnight at $37^{\circ} \mathrm{C}$ in a developing buffer containing $50 \mathrm{mM}$ Tris, $5 \mathrm{mM} \mathrm{CaCl}_{2} \cdot \mathrm{H}_{2} \mathrm{O}, 0.2 \mathrm{mM} \mathrm{NaCl}$, and $0.02 \%$ polyethylene glycol monododecyl ether (Brij-35; Tokyo Chemical Industry, Co, Ltd, Tokyo, Japan). Gels were stained for $30 \mathrm{~min}$ in $0.5 \%$ Coomassie blue R-250, $10 \%$ glacial acetic acid, and $10 \%$ isopropanol and de-stained in $50 \%$ methanol acid and $10 \%$ acetic acid for $10 \mathrm{~min}$, then dried and scanned.

\section{Statistical analysis}

Data are presented as mean \pm SEM. Statistical significance was assessed by one-way analysis of variance (ANOVA) with Turkey's multiple comparison test using GraphPad Prism Version 5.01 (GraphPad Software, Inc., La Jolla, CA). Statistical significance was defined as a value of $p<0.05$.

\section{Results}

\section{Effects of Apo on hemodynamics}

Hemodynamic parameters were measured at the end of the experiments. Our data showed that there was a significant difference in HR in AAC group as compared to the Sham group (Fig. $2 \mathrm{~A} ; p<0.05$ ). Neither captopril nor Apo treatment influenced HR (Fig. 2A). SAP was significantly increased in AAC group (Fig. 2B, $p<0.01$ ), and treatment with captopril but not apocynin reduced $\mathrm{SAP}$ in Cap group compared with AAC (Fig. 2B; $p<0.05$ ). Both LVSP and LVEDP were significantly increased in AAC group compared with Sham (Fig. 2C; $p<0.01)$. Captopril administration significantly reduced LVSP and LVEDP (Fig. 2C, D; $p<0.01$ ). Treatment with Apo only reduced LVEDP in Apo group (Fig. 2D; $p<0.05)$. Characteristic impairments in $\mathrm{dp} / \mathrm{dt}_{\max }$ and $\mathrm{dp} / \mathrm{dt}_{\min }$ were markedly increased in AAC (Fig. 2E, F; $p<0.01$ ) and only Apo markedly reduced $\mathrm{dp} / \mathrm{dt}_{\min }$ in Apo group (Fig. 2F; $p<0.05$ ).

\section{Effects of apocynin on interstitial fibrosis}

To evaluate interstitial fibrosis in heart tissue, collagen deposition was measured by Masson's trichrome staining. Compared with the Sham group, the interstitial fibrosis was significantly increased in AAC group. However, this increase was remarkably lower in the Cap and Apo groups (Fig. 3B, $p<0.01$ ).

\section{Effects of apocynin on expression of Ang II in serum and cardiac tissue}

The effects of apocynin and captopril on concentration of Ang II were assessed and results showed no significant difference in expression levels in the serum of all groups (Fig. 4A). However, the Ang II levels increased in cardiac tissue from the AAC group compared with the Sham group (Fig. 4B, $p<0.01$ ). Treatment with captopril but not apocynin had a significant effect on the levels of Ang II in cardiac tissue (Fig. 4B, $p<0.01$ ).

\section{Effects of apocynin on NADPH oxidase}

There was a significant increase in NADPH oxidase activity in the AAC group compared with the 
Sham group; however, its activity decreased in the presence of captopril and apocynin (Fig. 5A, $p<0.01$ ). Analysis of NADPH oxidase expression by western blot and RT-PCR demonstrated a significant increase in both protein and mRNA levels, respectively, for $\mathrm{p} 22^{\text {phox }}$ and $\mathrm{p} 67^{\text {phox }}$ in the AAC group compared to the Sham group (Fig. 5B-F, $p<0.05$ ). Treatment with captopril but not apocynin for 8 weeks had a significant effect on the levels of $\mathrm{p} 22^{\text {phox }}$ and p6 $7^{\text {phox }}$ (Fig. 5B-F, $p<0.05$ ).
A

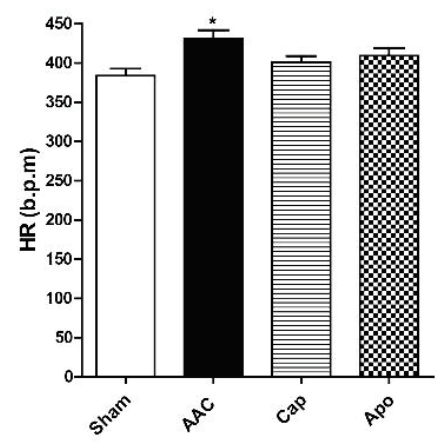

C

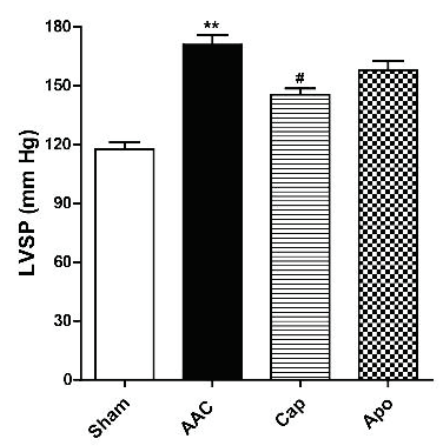

E
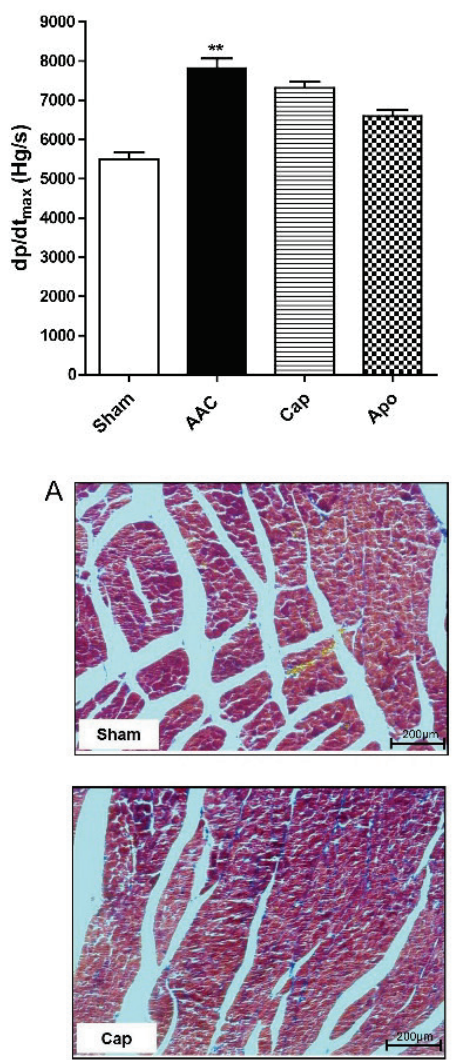

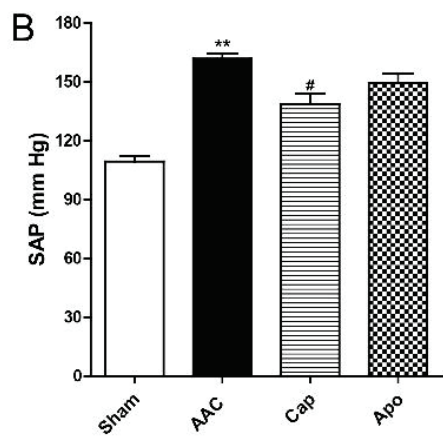

D
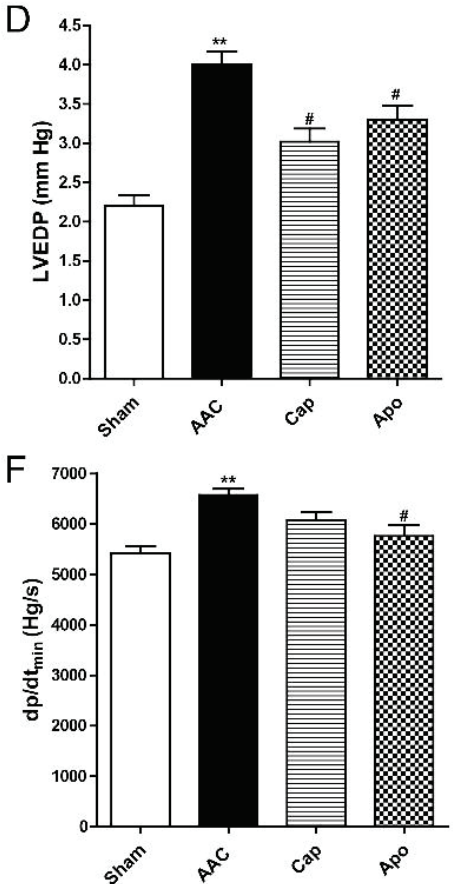

Fig. 2. Hemodynamic assessment of cardiac function after apocynin treatment in AAC group induced by pressure overload. Animals were anesthetized with pentobarbital sodium by intraperitoneal injection, and the right carotid arterial connected to pressure transducers was introduced into the left ventricle to measure HR (A), SAP (B), LVSP (C), LVEDP (D), $\mathrm{dp} / \mathrm{dt}_{\max }(\mathbf{E})$ and $\mathrm{dp} / \mathrm{dt}_{\min }(\mathbf{F})$ at 8 weeks after abdominal aorta constriction. Data are presented as mean \pm SEM, ${ }^{* *} p<0.01$ compared with Sham group, \#\# $p<0.01$ compared with AAC group. HR: heart rate, SAP: systolic arterial pressure, LVSP: left ventricular systolic pressure, LVEDP: left ventricular end-diastolic pressure, $\mathrm{dp} / \mathrm{dt}_{\max }$ and $\mathrm{dp} / \mathrm{dt}_{\min }$ : maximum/minimum values of the first derivative of left ventricular pressure.
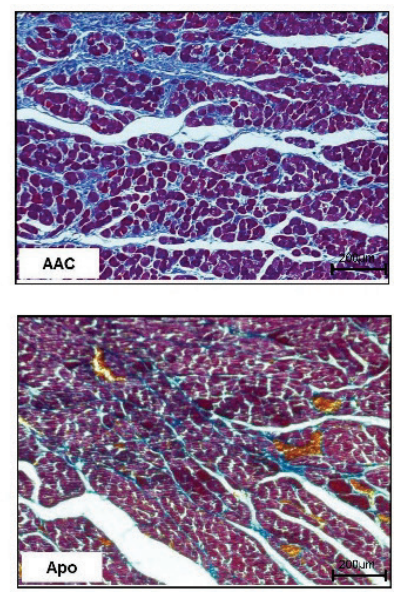

B

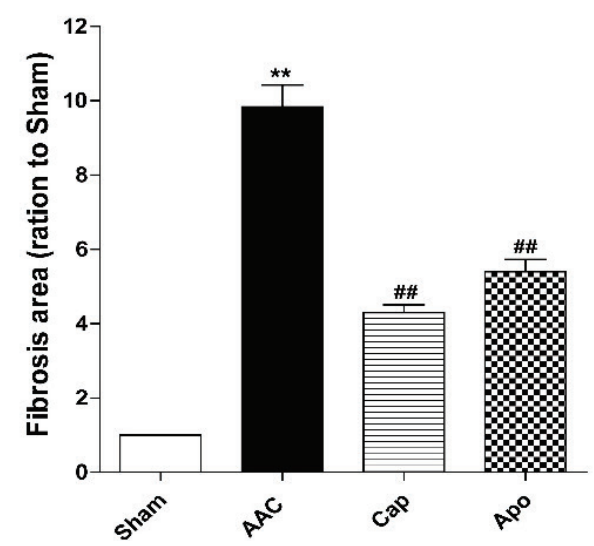

Fig. 3. Effects of apocynin on cardiac fibrosis induced by pressure overload in AAC group. (A) Representative collagen expression staining with Masson's trichrome and cardiac fibrosis was analyzed by morphometry via Masson's staining and visualized by light microscopy, scale bar $=200 \mu \mathrm{m}$. (B) Statistic results of myocardial fibrosis. Data are presented as mean \pm SEM, ${ }^{* *} p<0.01$ compared with Sham group, ${ }^{\# \#} p<0.01$ compared with AAC group. 
A
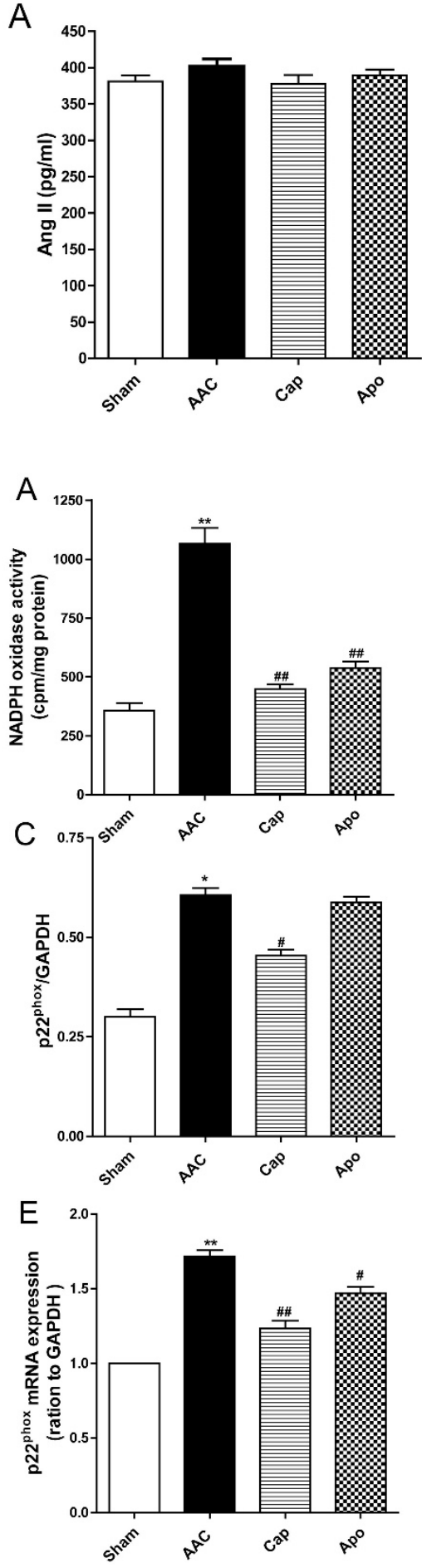

B

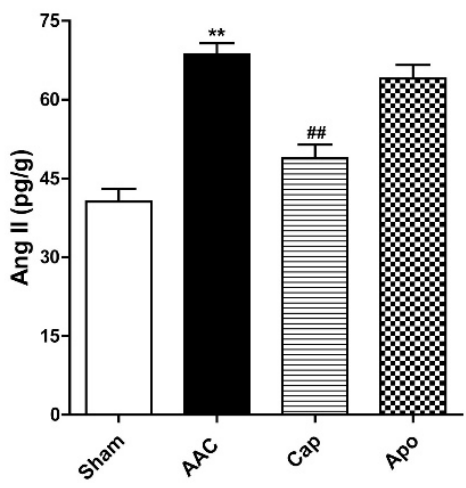

B
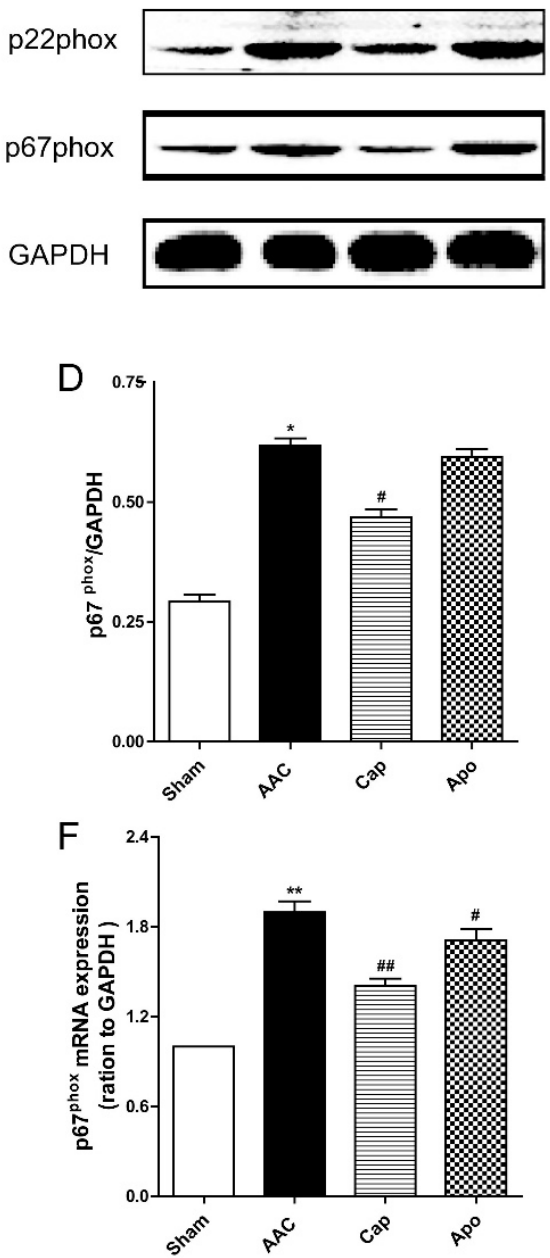

Fig. 4. Effects of apocynin on the levels of Ang II in serum and cardiac tissue. The level of Ang II in serum and cardiac tissue was measured by Radioimmunoassay. (A) Effect of apocynin on Ang II in serum. (B) Effect of apocynin on Ang II content assay in left ventricles. Data are presented as mean \pm SEM, ${ }^{* *} p<0.01$ compared with Sham group, ${ }^{\# \#} p<0.01$ compared with AAC group.

Fig. 5. Apocynin attenuates activity of $\mathrm{NADPH}$ oxidase in rats with pressure overload. (A) The activity of NADPH oxidase in left ventricle tissue were measured by colorimetrically analyzed. (B) Representative protein expression of p22 ${ }^{\text {phox }}$ and $\mathrm{p} 67^{\text {phox }}$ in left ventricles. (C, D) Statistic results of $\mathrm{p} 22^{\text {phox }}$ and $\mathrm{p} 67^{\text {phox }}(\mathbf{E}, \mathbf{F})$ The relative RNA expression level of $p 22^{\text {phox }}$ and $p 67^{\text {phox }}$ in left ventricles from rats were prepared and analyzed by RT-PCR. Data are presented as mean \pm SEM, ${ }^{*} p<0.05$ and ${ }^{* *} p<0.01$ compared with Sham group, \# $p<0.05$ and \#\# $p<0.01$ compared with AAC group.

\section{Effects of apocynin on ROS}

Superoxide anion levels were examined in cardiomyocytes using DHE staining. Our results showed that generation of superoxide anion was significantly elevated in cardiomyocytes from the AAC group compared with the Sham group (Fig. 6A, B, $p<0.01$ ). These changes were significantly reversed by captopril or apocynin administration (Fig. 6A, B, $p<0.05$ ). We further examined the effects of captopril and apocynin on MDA and SOD. The concentration of MDA was significantly higher in the AAC group compared with the Sham group (Fig. 6C, $p<0.01$ ). Treatment with captopril and apocynin significantly reduced the level of MDA (Fig. 6C, $p<0.01$ ). Conversely, the activity of SOD was significantly decreased in the AAC group compared with the Sham group. Treatment with captopril or apocynin increased SOD activity significantly, in the myocardium (Fig. 6D, $p<0.05)$. 

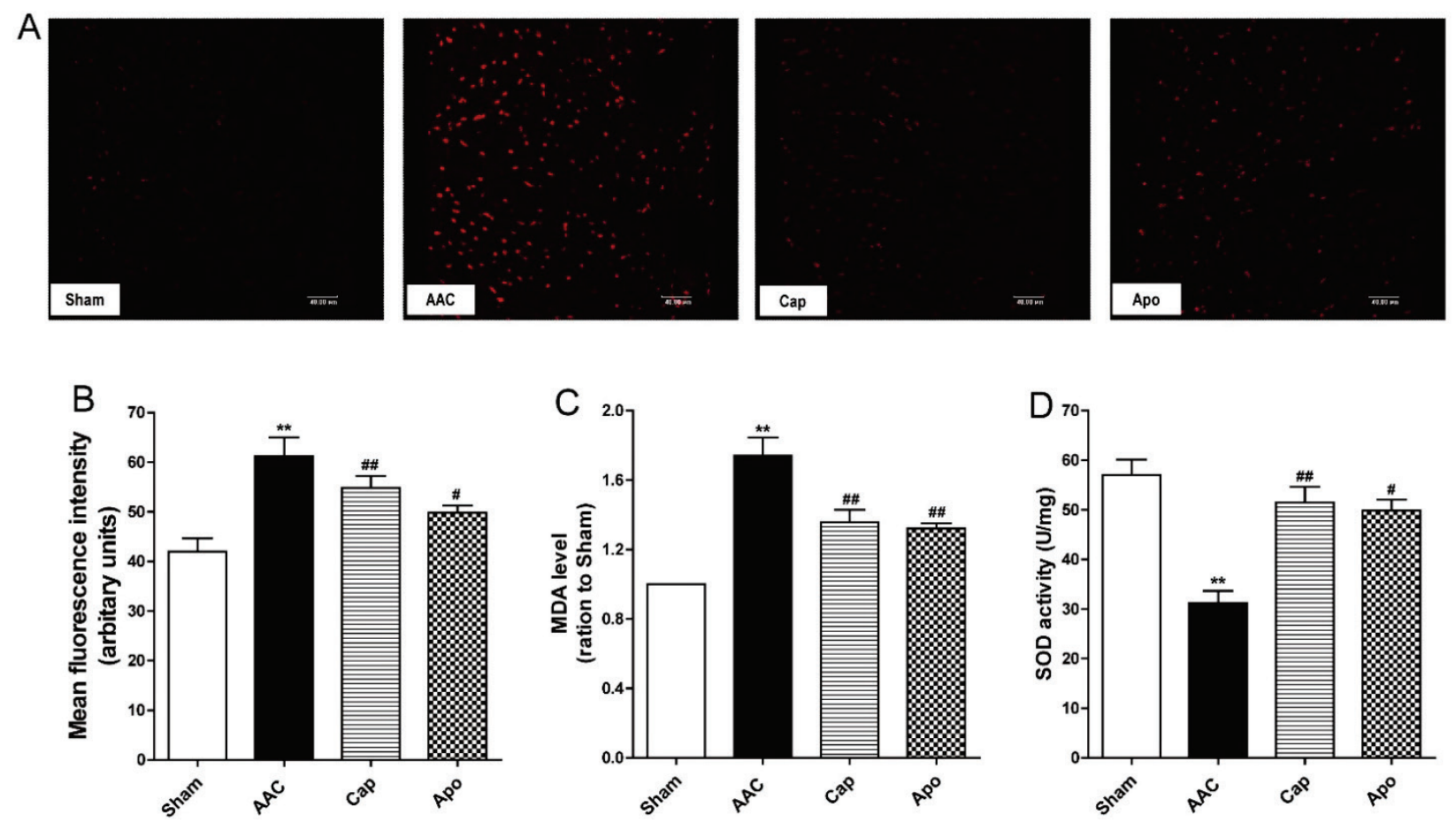

Fig. 6. Apocynin inhibits oxidative stress induced by pressure overload. (A) Representative images under fluorescence microscope, scale bar $=40 \mu \mathrm{m}$. (B) Statistic results of confocal microscope analysis of ROS level in left ventricles. (C, D) Activities of MDA and SOD in cardiac tissue of rats were colorimetrically analyzed using a microplate spectrophotometer and displayed. Data are presented as mean $\pm \mathrm{SEM}^{* *} p<0.01$ compared with Sham group, ${ }^{*} p<0.05$ and ${ }^{\# \#} p<0.01$ compared with AAC group. ROS: reactive oxygen species, MDA: Malondialdehyde, SOD: superoxide dismutase.

\section{A}
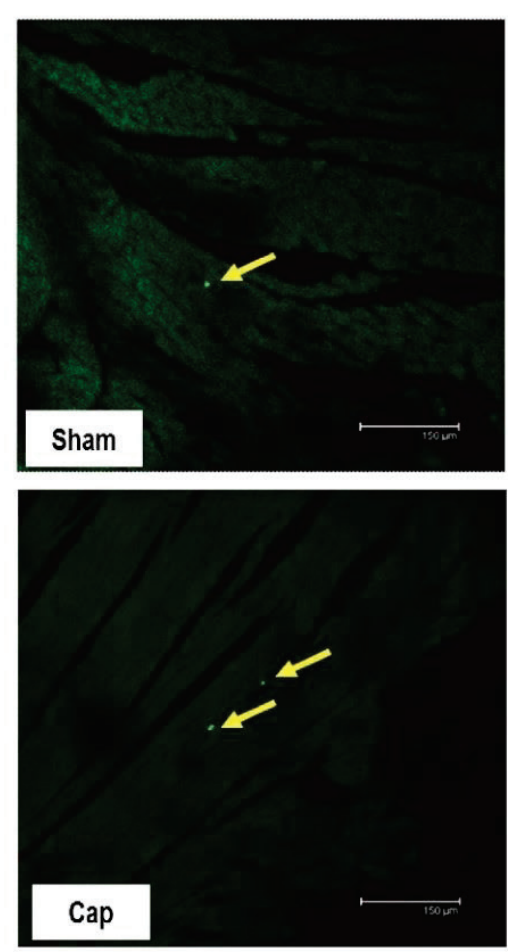
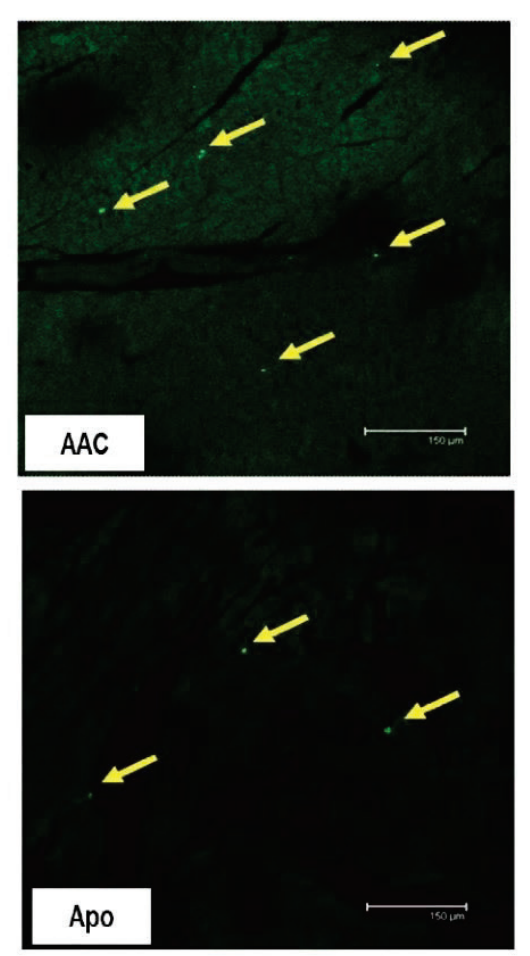
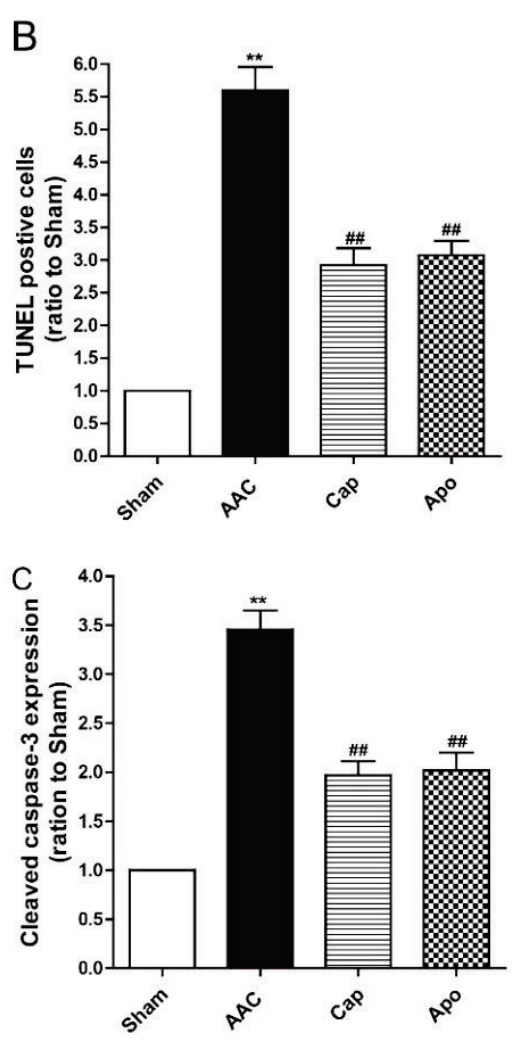

Fig. 7. Effects of apocynin on apoptotic index induced by pressure overload. (A) Representative TUNEL-positive left ventricular cardiomyocyte nuclei (green fluorescence, arrows) were counted under a fluorescent microscope, scale bar $=150 \mu \mathrm{m}$ (B) Quantitative result of TUNEL assay was analyzed in heart from all rats. (C) The level of caspase-3 in myocardial tissue was evaluated using a microplate spectrophotometer at $405 \mathrm{~nm}$ by the biochemical reagent kit. Data are presented as mean $\pm \mathrm{SEM},{ }^{* *} p<0.01 \mathrm{compared}$ with Sham group, ${ }^{\#} p<0.05$ and ${ }^{\# \#} p<0.01$ compared with AAC group. 
Apocynin reduced TUNEL staining and the expression of active caspase- 3 in cardiomyocytes

To study the effects of apocynin on cardiac apoptosis in myocytes, we evaluated the apoptotic index. TUNEL assay indicated that there was a significant increase in cardiomyocyte apoptosis in the AAC group (Fig. 7A, B, $p<0.01$ ). Treatment with captopril or apocynin significantly attenuated apoptosis compared with the AAC group (Fig. $7 \mathrm{~B}, p<0.01$ ). In line with the TUNEL staining, activity of caspase-3 was significantly increased in the AAC group compared with the Sham group (Fig. 7C, $p<0.01$ ). Moreover, administration of captopril or apocynin significantly reduced the activity of caspase-3 (Fig. 7C, $p<0.01$ ).

\section{Effects of apocynin on MMP-2}

MMP-2, a gelatinase that is crucial for degrading the extracellular matrix, has been implicated as an important factor in cardiac remodeling. We therefore investigated the expression and activity of MMP-2 in cardiac tissue using western blot and gelatin zymography. As shown in Fig. 8, the expression and activity of MMP-2 was significantly increased in the AAC group compared with the Sham group. Treatment with captopril or apocynin significantly reduced the expression and activity of MMP-2 in cardiac tissue (Fig. 8A-D, $p<0.01$ ).
A

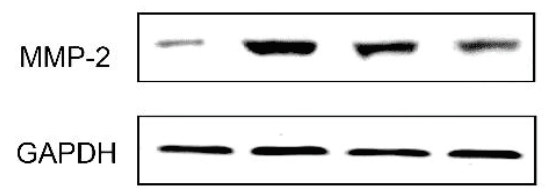

B

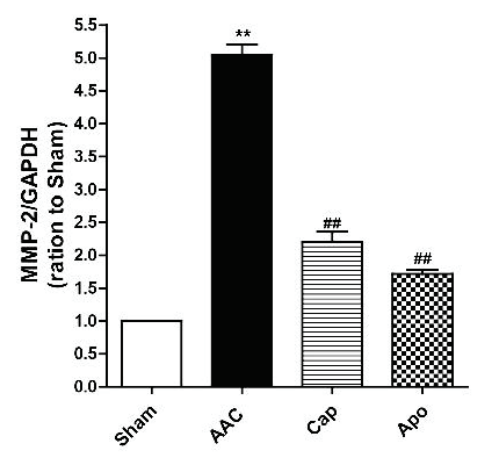

C

MMP-2

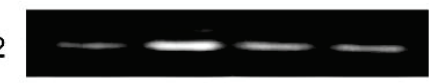

D

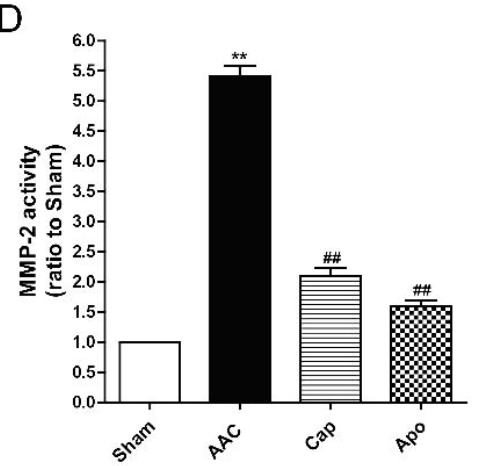

Fig. 8. Apocynin inhibits expression of MMP-2 in rats with pressure overload. The expression level of MMP-2 was assayed by Western blot (A, B) and gelatin zymography(C, D). Data are presented as mean \pm SEM, ${ }^{* *} p<0.01$ compared with Sham group, ${ }^{\# \#} p<0.01$ compared with AAC group. MMP-2: Metalloproteinase-2.

\section{Discussion}

In the present study, we examined the effects of apocynin on cardiac remodeling induced by pressure overload. We found that apocynin, the NADPH oxidase inhibitor, could attenuate cardiac remodeling by reducing the deposition of collagen, reducing the level of MMP-2 and by inhibiting up-regulated NADPH oxidase activity without affecting the expression of NADPH oxidase (p22 $2^{\text {phox }}$ and $\mathrm{p} 67^{\text {phox }}$ ). Additionally, apocynin ameliorated apoptosis in cardiomyocyte by suppressing the excessive production of ROS. Our results indicate that apocynin has cardioprotective effects, which might be related to its antioxidative effects.

Cardiac remodeling secondary to pressure overload is characterized by inappropriate hypertrophy, fibrosis, and apoptosis (Rain et al. 2014). RAS is a key regulator of pathophysiological processes and plays pivotal roles in cardiac remodeling, by accelerating fetal gene expression and collagen deposition. Ang II, a central biologically active downstream peptide of RAS, is directly involved in NADPH oxidase activation, oxidative stress, and cell apoptosis in myocardium during the pathogenesis of cardiac insult, and promotes unfavorable remodeling (Siddesha et al. 2013, Sumitomo-Ueda et al. 2010). Therefore, inhibiting the level of Ang II or ROS may as a pharmacological therapy to decrease cardiac remodeling and mortality in patients with HF. Reduction the level of Ang II by captopril, an ACEI, is used widely and highly effective in the treatment of HF through suppressing the activation RAS in clinical trials (Yoo et al. 2015). In addition, more and more studies from animal experiment further revealed that one of the protective mechanisms of captopril on 
CVD was closely related to the reduction of ROS (Kim et al. 2013, Kojsova et al. 2006). Apocynin is extracted from the root of Picrorhiza kurroa, which has been used as an efficient inhibitor of NADPH oxidase in many experimental models involving phagocytic and nonphagocytic cells. Apocynin has multiple protective effects on a variety of organs and tissues without interfering with other aspects of immune system (Chiang et al. 2011, Li et al. 2013, Li et al. 2012). Li et al. (2013) showed that apocynin suppresses myocardial oxidative stress and produces cardioprotective effects in Ang II-induced cardiac diastolic dysfunction in mice, which might be mediated partly through a pathway involving NADPH oxidase and osteopontin proteins. Our data showed that both apocynin and captopril ameliorate cardiac remodeling (Fig. 3). During this process, the level of Ang II in the left ventricle was reduced by captopril, but not apocynin (Fig. 4B). Additionally, the present study found no differences in serum Ang II levels in all groups (Fig. 4A). These results indicate that apocynin could attenuate cardiac remodeling independent of RAS.

It is well known that ROS derived from NADPH oxidase plays a critical role in the development and progression of cardiac remodeling (Yang et al. 2013, Taye et al. 2013). The inhibition of NADPH oxidase or reduction of superoxide anion generation represents an effective treatment for cardiac remodeling by reducing oxidative stress (Bai et al. 2013, Niu et al. 2012). The present study demonstrated that apocynin treatment could significantly inhibit the activity of NADPH oxidase (Fig. 5), reducing the superoxide anion production in the rat heart (Fig. 5A), and attenuating cardiac remodeling. These improvements were associated with a significant increase in SOD activity and decrease in MDA concentration (Fig. 6C, D). Therefore, these results further support that apocynin has obvious protective effect against cardiac remodeling induced by pressure overload, which might be related to its antioxidative effects.

A noticeable increase in cardiomyocyte apoptosis and a disproportionate accumulation of collagen, as well as MMPs in extracellular matrix were observed in rodents with pressure overload (Reddy et al. 2013). Furthermore, apoptosis resulting from oxidative stress plays an important role in cardiac remodeling, and inhibition or attenuation of cardiomyocyte apoptosis could prevent cardiac remodeling (Hill et al. 2013). Our findings suggest that apocynin provides significant antioxidative and anti-apoptotic effects (Fig. 7). These results imply that the beneficial effect of attenuating cardiomyocyte apoptosis mediated by apocynin might be related to inhibition of oxidative stress. MMPs have been viewed as potential and promising targets in CVD because of their key function as regulators of interstitial changes that degrade the collagen matrix (Brower et al. 2007). MMP-2, a member of the zinc-dependent proteinases, plays an important role in cardiac fibrosis (Molina et al. 2009). Some studies showed that MMP-2 was increased by NADPH oxidase overexpression at the mRNA, protein, and activity levels implying that, decreased oxidative stress and down-regulation of MMP-2 might prevent left ventricular structural remodeling (Hayashidani et al. 2002, Ceron et al. 2010, Liu et al. 2012a). In the present study, we found that apocynin significantly reduced MMP-2 levels in the AAC group and attenuated cardiac remodeling (Fig. 8). These results suggest that the effect of apocynin on MMP-2 may be related to its antioxidative effect.

Compared with captopril, apocynin plays an important role in inhibition the generation of ROS by suppressing directly the activity of NADPH oxidase without effect on the level of Ang II (Engels et al. 1992, Babior et al. 2002). The effect of apocynin on inhibition of ROS may bring us a new perspective for the treatment of cardiac remodeling.

In summary, the present study confirmed that apocynin reduced the production of ROS derived from NADPH oxidase, and inhibited disproportionate accumulation of collagen as well as cardiomyocyte apoptosis. Apocynin, however, had no effects on the level of Ang II in the left ventricular wall, which is involved in cardiac remodeling induced by pressure overload. Thus, apocynin markedly ameliorated cardiac remodeling, but this cardioprotective effect was independent of the RAS. The effect of apocynin on cardiac remodeling may form the basis for its use as therapeutic in CVD, and provide valuable information for CVD prevention and treatment.

\section{Conflict of Interest}

There is no conflict of interest.

\section{Acknowledgements}

This research was supported by National Natural Science Foundation of China (General program: Nos. 81471471 and 81000102) and Science and Technology Project of Shaanxi Province (No. 2012K17-02-08). 


\section{References}

AL-RASHEED NM, ATTIA HA, MOHAMED RA, AL-RASHEED NM, AL-AMIN M: Preventive effects of selenium yeast, chromium picolinate, zinc sulfate and their combination on oxidative stress, inflammation, impaired angiogenesis and atherogenesis in myocardial infarction in rats. J Pharm Pharm Sci 16: 848-867, 2013.

AL SUWAIDI J, AL-QAHTANI A, ASAAD N, AL-MULLA AW, SINGH R, ALBINALI HA: Comparison of women versus men hospitalized with heart failure (from a 20-year registry in a middle-eastern country 1991-2010). Am J Cardiol 109: 395-400, 2012.

BABIOR BM, LAMBETH JD, NAUSEEF W: The neutrophil NADPH oxidase. Arch Biochem Biophys 397: 342-344, 2002.

BAI J, ZHANG N, HUA Y, WANG BJ, LING L, FERRO A, XU B: Metformin inhibits angiotensin II-induced differentiation of cardiac fibroblasts into myofibroblasts. PLoS One 8: e72120, 2013.

BEDARD K, KRAUSE KH: The NOX family of ROS-generating NADPH oxidases: physiology and pathophysiology. Physiol Rev 87: 245-313, 2007.

BROWER GL, LEVICK SP, JANICKI JS: Inhibition of matrix metalloproteinase activity by ACE inhibitors prevents left ventricular remodeling in a rat model of heart failure. Am J Physiol Heart Circ Physiol 292: H3057-H3064, 2007.

CAI H, GRIENDLING KK, HARRISON DG: The vascular NAD(P)H oxidases as therapeutic targets in cardiovascular diseases. Trends Pharmacol Sci 24: 471-478, 2003.

CERON CS, CASTRO MM, RIZZI E, MONTENEGRO MF, FONTANA V, SALGADO MCO, GERLACH RF, TANUS-SANTOS JE: Spironolactone and hydrochlorothiazide exert antioxidant effects and reduce vascular matrix metalloproteinase-2 activity and expression in a model of renovascular hypertension. Brit J Pharmacol 160: 77-87, 2010.

CHEN BL, MA YD, MENG RS, XIONG ZJ, ZHANG CX, CHEN GQ, ZHANG AX, DONG YG: MG132, a proteasome inhibitor, attenuates pressure-overload-induced cardiac hypertrophy in rats by modulation of mitogen-activated protein kinase signals. Acta Bioch Bioph Sin 42: 253-258, 2010.

CHENG PW, HO WY, SU YT, LU PJ, CHEN BZ, CHENG WH, LU WH, SUN GC, YEH TC, HSIAO M, TSENG CJ: Resveratrol decreases fructose-induced oxidative stress, mediated by NADPH oxidase via an AMPK-dependent mechanism. Brit J Pharmacol 171: 2739-2750, 2014.

CHIANG CH, CHUANG CH, LIU SL: Apocynin attenuates ischemia-reperfusion lung injury in an isolated and perfused rat lung model. Transl Res 158: 17-29, 2011.

CHOUDHARY R, BAKER KM, PAN J: All-trans retinoic acid prevents angiotensin II- and mechanical stretch-induced reactive oxygen species generation and cardiomyocyte apoptosis. J Cell Physiol 215: 172-181, 2008.

CIARCIA R, DAMIANO S, PANICO C, SCANNI R, FIORITO F, FLORIO S, WELCH JW: Apocynin activity in spontaneously hypertensive rats (SHR): preliminary studies in vivo. Vet Res Commun 34 (Suppl 1): S83-S86, 2010.

COHN JN, FERRARI R, SHARPE N, REMODELING IFC: Cardiac remodeling-concepts and clinical implications: A consensus paper from an international forum on cardiac remodeling. J Am Coll Cardiol 35: 569-582, 2000.

COURTOIS E, MARQUES M, BARRIENTOS A, CASADO S, LOPEZ-FARRE A: Lead-induced downregulation of soluble guanylate cyclase in isolated rat aortic segments mediated by reactive oxygen species and cyclooxygenase-2. J Am Soc Nephrol 14: 1464-1470, 2003.

ENGELS F, RENIRIE BF, THART BA, LABADIE RP, NIJKAMP FP: Effects of apocynin, a drug isolated from the roots of Picrorhiza kurroa, on arachidonic-acid metabolism. FEBS Lett 305: 254-256, 1992.

GONZALEZ A, RAVASSA S, BEAUMONT J, LOPEZ B, DIEZ J: New targets to treat the structural remodeling of the myocardium. J Am Coll Cardiol 58: 1833-1843, 2011.

GREENBERG BH, ANAND IS, BURNETT JC, CHIN J, DRACUP KA, FELDMAN AM, FORCE T, FRANCIS GS, HOUSER SR, HUNT SA, ET AL.: The Heart Failure Society of America in 2020: a vision for the future. J Card Fail 18: 90-93, 2012. 
HASSAN F, MEDURU S, TAGUCHI K, KUPPUSAMY ML, MOSTAFA M, KUPPUSAMY P, KHAN M: Carvedilol enhances mesenchymal stem cell therapy for myocardial infarction via inhibition of caspase-3 expression. $J$ Pharmacol Exp Ther 343: 62-71, 2012.

HAYASHIDANI S, TSUTSUI H, SHIOMI T, SUEMATSU N, KINUGAWA S, IDE T, WEN J, TAKESHITA A: Fluvastatin, a 3-hydroxy-3-methylglutaryl coenzyme A reductase inhibitor, attenuates left ventricular remodeling and failure after experimental myocardial infarction. Circulation 105: 868-873, 2002.

HILL C, WURFEL A, HEGER J, MEYERING B, SCHLUTER KD, WEBER M, FERDINANDY P, ARONHEIM A, SCHULZ R, EULER G: Inhibition of AP-1 signaling by JDP2 overexpression protects cardiomyocytes against hypertrophy and apoptosis induction. Cardiovasc Res 99: 121-128, 2013.

JIN L, LAGODA G, LEITE R, WEBB RC, BURNETT AL: NADPH oxidase activation: a mechanism of hypertensionassociated erectile dysfunction. $J$ Sex Med 5: 544-551, 2008.

KIM JH, KIM H, KIM YH, CHUNG WS, SUH JK, KIM SJ: Antioxidant effect of captopril and enalapril on reactive oxygen species-induced endothelial dysfunction in the rabbit abdominal aorta. Korean J Thorac Cardiovasc Surg 46: 14-21, 2013.

KOJSOVA S, JENDEKOVA L, ZICHA J, KUNES J, ANDRIANTSITOHAINA R, PECHANOVA O: The effect of different antioxidants on nitric oxide production in hypertensive rats. Physiol Res 55 (Suppl 1): S3-S16, 2006.

LASSEGUE B, GRIENDLING KK: NADPH oxidases: functions and pathologies in the vasculature. Arterioscl Throm Vas 30: 653-661, 2010.

LI MC, LI ZA, WANG T, RAO K, YANG J, YANG J, QUAN WH, LIU JH, YE ZQ: Apocynin improves erectile function in diabetic rats through regulation of NADPH oxidase expression. J Sex Med 9: 3041-3050, 2012.

LI W, LUO Z, LIU X, FU L, XU Y, WU L, SHEN X: Effect of Ginkgo biloba extract on experimental cardiac remodeling. BMC Complement Altern Med 15: 277, 2015.

LI YQ, LI XB, GUO SJ, CHU SL, GAO PJ, ZHU DL, NIU WQ, JIA N: Apocynin attenuates oxidative stress and cardiac fibrosis in angiotensin II-induced cardiac diastolic dysfunction in mice. Acta Pharmacol Sin 34: 352-359, 2013.

LIU F, GARCIA AMG, MEYSKENS FL: NADPH Oxidase 1 overexpression enhances invasion via matrix metalloproteinase-2 and epithelial-mesenchymal transition in melanoma cells. J Invest Dermatol 132: 2033-2041, 2012a.

LIU JJ, ZHOU JA, AN WJ, LIN YX, YANG YB, ZANG WJ: Apocynin attenuates pressure overload-induced cardiac hypertrophy in rats by reducing levels of reactive oxygen species. Can J Physiol Pharm 88: 745-752, 2010.

LIU T, KORANTZOPOULOS P, LI G: Antioxidant therapies for the management of atrial fibrillation. Cardiovasc Diagn Ther 2: 298-307, 2012b.

MITRA A, BASAK T, DATTA K, NASKAR S, SENGUPTA S, SARKAR S: Role of alpha-crystallin B as a regulatory switch in modulating cardiomyocyte apoptosis by mitochondria or endoplasmic reticulum during cardiac hypertrophy and myocardial infarction. Cell Death Dis 4: e582, 2013.

MOLINA EJ, PALMA I, GUPTA D, TORRES D, GAUGHAN JP, HOUSER S, MACHA M: Reverse remodeling is associated with changes in extracellular matrix proteases and tissue inhibitors after mesenchymal stem cell (MSC) treatment of pressure overload hypertrophy. J Tissue Eng Regen Med 3: 85-91, 2009.

NAGALINGAM RS, SUNDARESAN NR, GUPTA MP, GEENEN DL, SOLARO RJ, GUPTA M: A cardiac-enriched microRNA, miR-378, blocks cardiac hypertrophy by targeting ras signaling. J Biol Chem 288: 11216-11232, 2013.

NIU X, WATTS VL, CINGOLANI OH, SIVAKUMARAN V, LEYTON-MANGE JS, ELLIS CL, MILLER KL, VANDEGAER K, BEDJA D, GABRIELSON KL, PAOLOCCI N, KASS DA, BAROUCH LA: Cardioprotective effect of beta-3 adrenergic receptor agonism: role of neuronal nitric oxide synthase. $J$ Am Coll Cardiol 59: 1979-1987, 2012.

RAIN S, HANDOKO ML, NOORDEGRAAF AV, BOGAARD HJ, VAN DER VELDEN J, DE MAN FS: Pressureoverload-induced right heart failure. Pflug Arch 466: 1055-1063, 2014.

REDDY S, ZHAO MM, HU DQ, FAJARDO G, KATZNELSON E, PUNN R, SPIN JM, CHAN FP, BERNSTEIN D: Physiologic and molecular characterization of a murine model of right ventricular volume overload. Am J Physiol Heart Circ Physiol 304: H1314-H1327, 2013. 
SCHWARZER M, OSTERHOLT M, LUNKENBEIN A, SCHREPPER A, AMORIM P, DOENST T: Mitochondrial reactive oxygen species production and respiratory complex activity in rats with pressure overload-induced heart failure. J Physiol-London 592: 3767-3782, 2014.

SIDDESHA JM, VALENTE AJ, SAKAMURI SSVP, YOSHIDA T, GARDNER JD, SOMANNA N, TAKAHASHI C, NODA M, CHANDRASEKAR B: Angiotensin II stimulates cardiac fibroblast migration via the differential regulation of matrixins and RECK. J Mol Cell Cardiol 65: 9-18, 2013.

SOVARI AA, MORITA N, KARAGUEUZIAN HS: Apocynin: a potent NADPH oxidase inhibitor for the management of atrial fibrillation. Redox Rep 13: 242-245, 2008.

ST JOHN SUTTON M, PFEFFER MA, MOYE L, PLAPPERT T, ROULEAU JL, LAMAS G, ROULEAU J, PARKER JO, ARNOLD MO, SUSSEX B, BRAUNWALD E: Cardiovascular death and left ventricular remodeling two years after myocardial infarction: baseline predictors and impact of long-term use of captopril: information from the Survival and Ventricular Enlargement (SAVE) trial. Circulation 96: 3294-3299, 1997.

SUMITOMO-UEDA Y, AIHARA K, ISE T, YOSHIDA S, IKEDA Y, UEMOTO R, YAGI S, IWASE T, ISHIKAWA K, HIRATA Y, AKAIKE M, SATA M, KATO S, MATSUMOTO T: Heparin cofactor II protects against angiotensin II-induced cardiac remodeling via attenuation of oxidative stress in mice. Hypertension 56: 430-436, 2010.

TAYE A, ABOUZIED MM, MOHAFEZ OMM: Tempol ameliorates cardiac fibrosis in streptozotocin-induced diabetic rats: role of oxidative stress in diabetic cardiomyopathy. Naunyn Schmiedebergs Arch Pharmacol 386: 1071-1080, 2013.

YANG CM, LEE IT, HSU RC, CHI PL, HSIAO LD: NADPH oxidase/ROS-dependent PYK2 activation is involved in TNF-alpha-induced matrix metalloproteinase-9 expression in rat heart-derived H9c2 cells. Toxicol Appl Pharm 272: 431-442, 2013.

YOO BS, PARK JJ, CHOI DJ, KANG SM, HWANG JJ, LIN SJ, WEN MS, ZHANG J, GE J: Prognostic value of hyponatremia in heart failure patients: an analysis of the Clinical Characteristics and Outcomes in the Relation with Serum Sodium Level in Asian Patients Hospitalized for Heart Failure (COAST) study. Korean J Intern Med 30: 460-470, 2015. 